\title{
A chimeric vector for dual use in cyanobacteria and Escherichia coli, tested with cystatin, a nonfluorescent reporter protein
}

\author{
Mojca Juteršek $^{1,2}$, Marko Dolinar ${ }^{\text {Corresp. } 1}$ \\ ${ }^{1}$ Faculty of Chemistry and Chemical Technology, University of Ljubljana, Ljubljana, Slovenia \\ 2 National Institute of Biology, Ljubljana, Slovenia \\ Corresponding Author: Marko Dolinar \\ Email address: marko.dolinar@fkkt.uni-lj.si
}

\section{Background.}

Developing sustainable autotrophic cell factories depends heavily on the availability of robust and wellcharacterized biological parts. For cyanobacteria, these still lag behind the more advanced E. coli toolkit. In the course of previous protein expression experiments with cyanobacteria, we encountered inconveniences in working with currently available RSF1010-based shuttle plasmids, particularly due to their low biosafety and low yields of recombinant proteins. We also recognized some drawbacks of the commonly used fluorescent reporters, as quantification can be affected by the intrinsic fluorescence of cyanobacteria. To overcome these drawbacks, we envisioned a new chimeric vector and an alternative reporter that could be used in cyanobacterial synthetic biology and tested them in the model cyanobacterium Synechocystis sp. PCC 6803.

\section{Methods.}

We designed the pMJc01 shuttle plasmid based on the broad host range RSFmob-I replicon. Standard cloning techniques were used for vector construction following the RFC10 synthetic biology standard. The behavior of pMJC01 was tested with selected regulatory elements in E. coli and Synechocystis sp. PCC 6803 for the biosynthesis of the established GFP reporter and of a new reporter protein, cystatin. Cystatin activity was assayed using papain as a cognate target.

\section{Results.}

With the new vector we observed a significantly higher GFP expression in E. coli and Synechocystis sp. PCC 6803 compared to the commonly used RSF1010-based pPMQAK1. Cystatin, a cysteine protease inhibitor, was successfully expressed with the new vector in both E. coli and Synechocystis sp. PCC 6803. Its expression levels allowed quantification comparable to the standardly used fluorescent reporter GFPmut3b. An important advantage of the new vector is its improved biosafety due to the absence of plasmid regions encoding conjugative transfer components. The broad-host range vector pMJc01 could find application in synthetic biology and biotechnology of cyanobacteria due to its relatively small size, stability and ease of use. In addition, cystatin could be a useful reporter in all cell systems that do not contain papain-type proteases and inhibitors, such as cyanobacteria, and provides an alternative to fluorescent reporters or complements them. 
1 A chimeric vector for dual use in cyanobacteria and Escherichia coli, tested

2 with cystatin, a nonfluorescent reporter protein

3 Mojca Juteršek ${ }^{1,2}$ and Marko Dolinar ${ }^{1}$

4

$5 \quad{ }^{1}$ University of Ljubljana, Faculty of Chemistry and Chemical Technology, Ljubljana, Slovenia

$6 \quad{ }^{2}$ Current address: National Institute of Biology, Ljubljana, Slovenia

7

8 Corresponding Author:

9 Marko Dolinar ${ }^{1}$

10

11 Email address: marko.dolinar@fkkt.uni-lj.si

12 


\section{Abstract}

14

\section{Background.}

Developing sustainable autotrophic cell factories depends heavily on the availability of robust and well-characterized biological parts. For cyanobacteria, these still lag behind the more advanced $E$. coli toolkit. In the course of previous protein expression experiments with cyanobacteria, we encountered inconveniences in working with currently available RSF1010based shuttle plasmids, particularly due to their low biosafety and low yields of recombinant proteins. We also recognized some drawbacks of the commonly used fluorescent reporters, as quantification can be affected by the intrinsic fluorescence of cyanobacteria. To overcome these drawbacks, we envisioned a new chimeric vector and an alternative reporter that could be used in cyanobacterial synthetic biology and tested them in the model cyanobacterium Synechocystis sp. PCC 6803.

\section{Methods.}

We designed the pMJc01 shuttle plasmid based on the broad host range RSFmob-I replicon. Standard cloning techniques were used for vector construction following the RFC10 synthetic biology standard. The behavior of pMJC01 was tested with selected regulatory elements in $E$. coli and Synechocystis sp. PCC 6803 for the biosynthesis of the established GFP reporter and of a new reporter protein, cystatin. Cystatin activity was assayed using papain as a cognate target.

\section{Results.}

With the new vector we observed a significantly higher GFP expression in E. coli and Synechocystis sp. PCC 6803 compared to the commonly used RSF1010-based pPMQAK1. 
34 Cystatin, a cysteine protease inhibitor, was successfully expressed with the new vector in both $E$.

35 coli and Synechocystis sp. PCC 6803. Its expression levels allowed quantification comparable to

36 the standardly used fluorescent reporter GFPmut3b. An important advantage of the new vector is

37 its improved biosafety due to the absence of plasmid regions encoding conjugative transfer

38 components. The broad-host range vector pMJc01 could find application in synthetic biology

39 and biotechnology of cyanobacteria due to its relatively small size, stability and ease of use. In

40 addition, cystatin could be a useful reporter in all cell systems that do not contain papain-type

41 proteases and inhibitors, such as cyanobacteria, and provides an alternative to fluorescent

42 reporters or complements them. 


\section{Introduction}

44 Cyanobacteria are emerging as versatile hosts for synthetic biology. Starting as poorly adapted

45 microorganisms for genetic engineering, advances in the last decade have made them attractive 46 organisms for biosynthetic production of commodities ranging from biofuels to high added-value

47 compounds. A book (Zhang \& Song, 2018), several book chapters (e.g., Klemenčič et al. 2017;

48 Badary \& Sode, 2020; Hudson, 2021) and review articles (Khan et al., 2019; Lindblad et al.,

49 2019; Santos-Merino, Singh \& Ducat, 2019; Vavitsas et al., 2019; Ng, Keskin \& Tan, 2020;

50 Wang, Gao \& Yang, 2020; Liu et al., 2021; Sproles et al., 2021) published in recent years

51 emphasize the potential of cyanobacteria and present encouraging results obtained with these

52 hosts.

53 To realize their full application potential as green engineered biofactories, a set of well-

54 characterized genetic elements is necessary to provide a starting material for higher-order

55 synthetic systems. Although the arsenal of available and characterized regulatory sequences for

56 cyanobacteria is expanding, there is still an urgent need to develop stronger and better regulated

57 promoters, ribosome binding sites and terminators. For this, we need reliable reporter systems

58 that allow quantification of the effects achieved by novel genetic elements, and a reliable

59 plasmid backbone.

60 Fluorescent proteins are most commonly used as reporters in cyanobacteria because they allow

61 both high-throughput analyses and in vivo measurements. Despite their clear advantages and

62 popularity, some properties make them less suitable for certain experimental setups. Fluorophore

63 formation is oxygen-dependent and reporter activity can therefore vary with oxygen levels in the

64 cell. In addition, fluorescent proteins can have problems with sensitivity due to the lack of signal 
amplification and background autofluorescence. This is particularly critical in cyanobacteria with strong intrinsic fluorescence from photosynthetic pigments, which can also act as fluorescence quenchers (Taniuchi, Murakami \& Ohki, 2008). An alternative reporter system based on a different measurement technique could counteract the disadvantages of fluorescence reporters and improve the reliability of the measurements, thus contributing to a better characterization of biological parts.

Essential to modern cyanobacterial biotechnology is the introduction of new genetic elements into host strains. There are two general approaches to introduce such elements into cyanobacteria: genomic insertion and episomal introduction. The former is complicated by a limited number of true neutral sites in the genome (Pinto et al., 2015) and by a low number of genome copies. Where multiple copies of the genome exist, as in the best-studied unicellular cyanobacterium Synechocystis sp. PCC 6803 (Zerulla, Ludt \& Soppa, 2016), obtaining strains in which all genomic copies are genetically modified is time-consuming and requires quantitative analyses (Hitchcock, Hunter \& Canniffe, 2020). Therefore, episomal expression is often preferred, especially for characterization of biological parts and protein expression. Although many vectors have been developed, they are not generally suitable to every experimental setup.

The first group of cyanobacterial vectors is based on replicators from endogenous cyanobacterial plasmids that lack broad host range fitness. This can be overcome by using RSF1010-based plasmids, but their biological safety is questionable (Wright, Stan \& Ellis, 2013), as they allow conjugative transfer; mobilization is possible by a variety of different type IV transporters (Meyer, 2009). In addition, RSF1010-derived plasmids have low copy number in both E. coli and cyanobacteria. In E. coli, copy numbers are probably 5 or even less (Jahn et al., 2016), although 10-12 copies per chromosome copy were originally reported (Frey and Bagdasarian 
88 1989). Similarly low copy numbers have been reported in cyanobacteria: 10 copies per cell in

89 Synechocystis sp. PCC 6803 and PCC 6714 and Synechococcus sp. PCC 7942 (Mermet-Bouvier

90 et al., 1993) or one plasmid copy per genome copy for pPMQAK1 vectors in Synechocystis sp.

91 PCC 6803 (Huang et al., 2010; Jin et al., 2018).

92 Low plasmid copy number could be reflected in lower protein expression levels compared to

93 high copy number plasmids. The correlation between plasmid copy number and reporter

94 expression was clearly demonstrated by Jahn et al. (Jahn et al., 2016) for RSF1010-based

95 plasmids in E. coli, where two subpopulations of cells with different levels of EGFP expression

96 were detected. A similar observation was reported by Thompson et al. (Thompson et al., 2018)

97 on pSC101 ori mutants. Such correlation has also been observed in other bacteria, e.g.

98 Corynebacterium glutamicum (Hashiro \& Yasueda, 2018), while quantitative data for

99 cyanobacterial hosts are still lacking. A difference in vector copy number was suspected for

100 differences in reporter expression in Anabaena sp. PCC 7120 (Ma, Schmidt \& Golden, 2014),

101 while in Synechococcus elongatus PCC 7942, reporter expression level correlated well with

102 vector copy number (Chen et al., 2016).

103 Conjugation is a process in which bacteria exchange their genetic material and in which plasmids

104 act as vectors. Although conjugation is commonly used to introduce plasmid vectors into

105 cyanobacterial cells, usually by exploiting the so-called triparental mating (Vioque, 2007),

106 cyanobacteria can also be transformed by electroporation and some strains are even naturally

107 competent for transformation (Zang et al., 2007). In our experiments with the model

108 cyanobacterium Synechocystis sp. PCC 6803, we use electroporation exclusively. Therefore, we

109 could easily trade off the conjugation-related genetic elements on the vector for improved

110 biosafety and smaller size.

Peer] reviewing PDF | (2021:06:62918:1:1:NEW 27 Aug 2021) 
111 Our goal was to (a) construct a shuttle vector with increased copy number and improved

112 biological safety and (b) present a reporter that is independent of intrinsic fluorescence or other

113 activity in cyanobacteria. For the vector, we chose the pPMQAK1 plasmid as a starting point for

114 modifications. This vector is a commonly used RSF1010-based shuttle plasmid for

115 cyanobacterial synthetic biology and has been used in our lab for several years. However, to

116 improve its biological safety, we wanted to retain regions that are required for replication in

117 different bacterial hosts, but not those that enable conjugative transfer (oriT and mob regions).

118 Therefore, we decided to complement selected pPMQAK1 regions with those of RSFmob-I, a

119 non-mobilizable RSF1010 derivative developed by Katashkina and coworkers (Katashkina et al.,

120 2007). We inserted into the new vector the kanamycin resistance gene and a standardized

121 synthetic biology cloning site compatible with RFC10 assembly (Knight, 2003; Shetty, Endy \&

122 Knight, 2008).

123 The constructed pMJc01 vector backbone was tested and characterized in both E. coli and

124 Synechocystis sp. PCC 6803. First, the common GFPmut3b fluorescent reporter (Cormack,

125 Valdivia \& Falkow, 1996) was used to demonstrate the usability of the vector. Then, we used the

126 improved vector backbone to investigate the conformity of the vector with cystatin as a new non-

127 fluorogenic reporter. Cystatin is a stable protein first described as a hen egg-white protease

128 inhibitor (Fossum \& Whitaker 1968). We chose cystatin (reviewed in Turk, Stoka \& Turk, 2008)

129 as a potential reporter because it can be easily assayed with an enzyme inhibition test using

130 papain (a cysteine protease) as the target enzyme. The enzyme-based detection method also

131 allows for signal amplification and thus higher sensitivity. We found cystatin to be a useful

132 reporter both in E. coli and in Synechocystis sp. PCC 6803. It could be used as an alternative or

133 to complement fluorescent protein reporters. 


\section{Materials and Methods}

\section{Strains and growth conditions}

137 Cultures of the cyanobacterium Synechocystis sp. PCC 6803 (S. sp. PCC 6803, obtained from

138 Pasteur Culture Collection of Cyanobacteria) were grown on BG11 agar plates or in BG11 liquid

139 medium (Stanier et al., 1971) at room temperature under continuous white light of 10-30 $\mu \mathrm{mol}$

140 photons $\mathrm{m}^{-2} \mathrm{~s}^{-1}$. Liquid cultures were shaken at $100 \mathrm{rpm}$. For engineered strains transformed with

141 pMJc01 or pPMQAK1 plasmid or their derivatives, the growth medium was supplemented with

$14250 \mu \mathrm{g} / \mathrm{ml}$ kanamycin.

143 Escherichia coli XL1-Blue was used for cloning and characterization of vector constructs. All

144 strains were cultured in liquid LB medium with shaking at $140 \mathrm{rpm}$ at $37^{\circ} \mathrm{C}$ or on LB agar plates.

145 Cells transformed with pMJc01, pSB3K3 or pPMQAK1 were grown in a medium containing 50

$146 \mu \mathrm{g} / \mathrm{ml}$ kanamycin and those transformed with the RSFmob-I plasmid were cultured in the

147 presence of $50 \mu \mathrm{g} / \mathrm{ml}$ streptomycin.

148 For the transformation of E. coli, we used chemically competent cells and for the transformation

149 of $S$. sp. PCC 6803, we used electroporation. In brief, cells in the exponential growth phase were

150 washed three times in ice-cold sterile Milli-Q water (followed by centrifugation for $10 \mathrm{~min}$ at

$1514^{\circ} \mathrm{C}$ and $6000 \mathrm{~g}$ ) and mixed with $0.5-2 \mu \mathrm{g}$ of plasmid dissolved in $40 \mu \mathrm{l}$ of sterile Milli-Q water.

152 The suspension of cells and plasmid was transferred to electroporation cuvettes with a gap width

153 of $2 \mathrm{~mm}$ and subjected to an electrical pulse of $2500 \mathrm{~V}$ for $5 \mathrm{~ms}$ (with the capacitance set to 25

$154 \mu \mathrm{F}$ and the resistance set to $200 \Omega$ ). After pulse treatment, cells were immediately resuspended in

$1555 \mathrm{ml}$ of BG11 medium without kanamycin and left to recover overnight at lower light intensity. 
156 The next day, the liquid cultures were poured onto BG11 agar plates supplemented with $5 \mu \mathrm{g} / \mathrm{ml}$

157 kanamycin. The resulting colonies were transferred to BG11 agar plates supplemented with

158 gradually increasing concentrations of kanamycin (10, 25 and finally $50 \mu \mathrm{g} / \mathrm{ml})$.

\section{Construction of the broad host range shuttle vector pMJc01}

160 A DNA region comprising sequences required for autonomous self-replication was PCR-

161 amplified with primers RSFmobF and RSFmobR (Table 1) from the template plasmid RSFmob-I

162 (Katashkina et al., 2007) (available via Russian National Collection of Industrial

163 Microorganisms). A DNA fragment containing a synthetic biology RFC[10] standard cloning

164 site and a kanamycin resistance cassette was PCR-amplified from the pSB3K3 template plasmid 165 using primers pSB3K3F and pSB3K3R (Table 1). Primers RSFmobR and pSB3K3F contained 166 an 18 bp long complementary sequence that allowed overlap extension PCR for splicing of the

167 3771-bp fragment from plasmid RSFmob-I and the 2238-bp fragment from plasmid pSB3K3 to 168 plasmid pMJc01. The corresponding linear 5999-bp product was phosphorylated at the 5 ' ends, 169 circularized with T4 DNA ligase, and used to transform competent $E$. coli cells. The sequence of 170 the new pMJc01 vector was verified by Sanger sequencing (GATC Biotech, Eurofins 171 Genomics).

172 Construction of GFPmut3b or cystatin reporter-expressing plasmids based on pMJc01, 173 pPMQAK1 and pSB3K3

174 For comparative characterization of the new vector and evaluation of cystatin as a reporter, we 175 constructed plasmids pMJc01, pPMQAK1, and pSB3K3 without reporter-expressing sequences

176 (EV) and with 3 different expression cassettes (Table 2). For the first cassette, we chose

177 BBa_I20260 (all BBa codes refer to the Registry of Standard Biological Parts, 
178 http://parts.igem.org/Main_Page, from which they can be retrieved as sequences or as physical 179 DNA inserted into a plasmid backbone), a reference standard construct for relative promoter 180 activity measurements based on GFPmut3b (Kelly et al., 2009). Plasmids pMJc01 and pSB3K3 181 lacking BBa_I20260 were obtained by XbaI/SpeI restriction followed by ligation. For plasmid 182 pPMQAK1, we started from the pPMQAK1_EV plasmid (BBa_J153000), which was previously 183 prepared in our laboratory. To make the pPMQAK1_BBa_I20260 plasmid, we cut the 184 pPMQAK1_EV backbone with EcoRI and PstI. The same restriction endonucleases were used to 185 excise BBa_I20260 from pMJc01 and clone it into the pPMQAK1 plasmid backbone.

186 Since the regulatory sequences in BBa_I20260 (BBa_J23101 promoter and BBa_B0032 RBS) 187 show low activity in Synechocystis sp. PCC 6803 (Huang \& Lindblad, 2013; Englund, Liang \& 188 Lindberg, 2016), we additionally prepared reporter constructs under the control of the 189 L21_RBS*regulatory element. The designed regulatory element was made from the 190 complementary oligonucleotides L21_RBS*_F and L21_RBS*_R and comprises the -35 element (5'-TTGACA-3'), the -10 element (5'-TATAAT-3'), and two tetO2 operators: one upstream of the -35 element and one between the -35 and -10 elements (Table 1). We chose L21 because the expression of the reporter in Synechocystis was about 10-fold stronger compared to J23101 and because it did not respond to anhydrotetracyline, which was desirable in our case because we were looking for a constitutive reporter.

The coding sequence of the cystatin reporter was designed based on the amino acid sequence deposited in the UniProt database (chicken cystatin, accession number P01038). We included residues 24-139, which constitute a functional protein with 2 disulfide bridges, and reversetranslated them into the nucleotide sequence. An $X b a \mathrm{I}$ restriction site with start codon $\left(5^{\prime}-\right.$ 
201 sites was added to the 3' end of the cystatin coding sequence (cystatin6803). The sequence was

202 codon-optimized and synthesized by Synbio Technologies.

203 The GFPmut3b coding region was PCR-amplified from a recombinant pSB3K3 derivative with

204 primers GFPmut3bF and VR (Table 1) and cut with $X b a \mathrm{I} / P s t \mathrm{I}$. The same restriction

205 endonucleases were used to cut cystatin6803 inserted by the supplier into the pUC57 plasmid.

206 Complementary oligonucleotides containing the regulatory sequence L21_RBS* were hybridized

207 and cut with SpeI. The reporter coding regions and the regulatory fragment were ligated with

208 complementary $X b a \mathrm{I} / S p e \mathrm{I}$ overhangs and the product was cut with $X b a \mathrm{I}$. The obtained reporter

209 gene expressing cassettes were inserted into plasmid backbones pSB3K3, pMJc01, and

210 pPMQAK1, which were previously linearized with $X b a \mathrm{I} / P s t \mathrm{I}$ restriction endonucleases. E. coli

211 cells were transformed with the constructed plasmids and colonies were verified by colony PCR

212 using primers VF2 and VR after overnight incubation (Table 1).

213 Characterization of the pMJc01 vector in $E$. coli

214 The relative reporter expression from $\mathrm{pMJc01}, \mathrm{pSB} 3 \mathrm{~K} 3$, and pPMQAK1 in E. coli was

215 determined using GFPmut3b fluorescence at the stationary growth phase in cell lysates and in

216 liquid cultures (similarly to Huang et al., 2010). For measurements in liquid cultures, overnight

217 cultures of cells transformed with each of the three plasmid backbones without insert and with

218 BBa_I20260 or L21_RBS*_GFPmut3b reporter constructs were diluted 8-fold to the final

219 volume of $2 \mathrm{ml}$ in LB medium, supplemented with kanamycin (final $\mathrm{Abs}_{600} 0.10-0.14$ ).

220 Alternatively, for measurements of cell lysates, cells were pelleted from $2 \mathrm{ml}$ overnight cultures

221 and resuspended in TE (10 mM Tris $\mathrm{pH} 8.0,1 \mathrm{mM}$ EDTA) buffer, with volume adjusted to

222 achieve equal cell densities in all cell suspensions. Cells were lysed by sonication for $3 \times 10 \mathrm{~s}$ 
223 and $35 \mu \mathrm{l}$ of the cell lysate was resuspended in $2 \mathrm{ml} \mathrm{TE}$ buffer. Fluorescence emission spectra

$224(505-530 \mathrm{~nm})$ were measured in glass cuvettes with $1 \mathrm{~cm}$ light path in Perkin Elmer LS50B

225 fluorimeter with the excitation wavelength of $485 \mathrm{~nm}$. The average of five spectra was recorded

226 for each sample and the fluorescence intensity at the emission maximum (518 nm for cultures

227 and $514.5 \mathrm{~nm}$ for cell lysates) was read. The values of fluorescence intensity in the cultures were

228 normalized to cell density $\left(\mathrm{F} / \mathrm{Abs}_{600}\right)$ and the average $\mathrm{F} / \mathrm{Abs}_{600}$ ratio of the biological triplicates

229 of the samples with empty plasmids was subtracted from the F/Abs 600 ratios of the biological

230 triplicates of the same plasmid with reporter construct and the average was calculated from the

231 differences. For cell lysates, the fluorescence intensity value of cells with empty plasmid was

232 subtracted from the intensity values of the triplicates of plasmids with reporter constructs and the

233 average was calculated from the differences.

234 Quantification of restriction fragments in the agarose gel was used to evaluate the absolute copy

235 numbers of pMJc01, pPMQAK1, and pSB3K3. This approach to plasmid copy number

236 determination is based on the established densitometric technique described by Projan et al.

237 (Projan, Carleton \& Novick, 1983), but we adapted it for comparison of plasmids with different

238 sizes so that only the fluorescence of a restriction fragment of the same size was quantified in all

239 the plasmids. We isolated pMJc01, pPMQAK1, and pSB3K3 plasmids with the

240 L21_RBS*_GFPmut $3 b$ insert from approximately $5 \times 10^{9}$ E. coli cells from overnight cultures

241 and used NotI for digestion, resulting in linear plasmid backbones and a 943-bp fragment

242 encoding the reporter. Restriction products were loaded alongside various amounts of DNA

243 standards (100 bp DNA ladder from New England Biolabs and GeneRuler $1 \mathrm{~kb}$ DNA ladder

244 from Thermo Scientific), separated in a 1.5\% agarose gel with ethidium bromide, and visualized

245 under UV light. The gel image was analyzed using GelAnalyzer software, and the 943-bp 
246 fragment was quantified using a calibration curve generated with known amounts of 1000-bp

247 fragments in the ladders used. For $\mathrm{pMJc0} 1$ and $\mathrm{pSB} 3 \mathrm{~K} 3$, we also performed restriction with

248 BspHI, resulting in a 2128-bp fragment from both plasmids, and used known amounts of the

249 2000-bp marker for quantification.

250 Measurements of GFPmut3b fluorescence at different growth stages were performed on E. coli

251 transformed with pMJc01_L21_RBS*_GFPmut3b and pMJc01_EV. We took culture samples

252 from the liquid cultures (grown in biological triplicates) at different time points and diluted them

253 to $\mathrm{Abs}_{600}$ of 0.1 . Fluorescence measurements were performed as described above. Since all

254 samples were diluted to equal cell densities, no normalization of fluorescence values was

255 performed.

256 Characterization of cystatin reporter in $E$. coli

257 To assess the expression of recombinant chicken cystatin in E. coli, we used an adapted papain

258 inhibition assay (Kopitar et al., 1978). After overnight cultivation, cells were pelleted from 1.5

$259 \mathrm{ml}$ of bacterial culture and suspended in TE buffer, with the volume adjusted to achieve equal

260 cell densities in all samples. Cells were lysed by sonication for $3 \times 10 \mathrm{~s}$ and lysates were

261 centrifuged at $14000 \mathrm{~g}$ for $2 \mathrm{~min}$. For the inhibition assay, $35 \mu 1$ of the soluble fraction was used

262 together with $175 \mu \mathrm{l}$ of $100 \mathrm{mM}$ phosphate buffer (pH 6.0), $105 \mu 1$ of TE buffer and $35 \mu 1$ of

263 papain solution $(35 \mu \mathrm{g} / \mathrm{ml})$ in phosphate buffer. Two controls were used: a blank without enzyme

264 to determine background absorbance (used to set zero absorbance on the spectrophotometer) and

265 a positive control without cell lysate to confirm the enzymatic activity of the papain used. After

266 incubation of the samples at $37^{\circ} \mathrm{C}$ for 5 minutes, $35 \mu 1$ of the substrate BANA (N-benzoyl- DL -

267 arginine naphthylamide hydrochloride) was added $(6.7 \mathrm{mg} / \mathrm{ml}$, freshly diluted 1:5 in phosphate 
268 buffer from DMSO stock solution) and incubated at $37^{\circ} \mathrm{C}$ for a further 10 minutes. The enzyme

269 reaction was terminated by adding $385 \mu 1$ of a 1:1 mixture of Fast Garnet solution in 4\% Brij $(0.3$

$270 \mathrm{mg} / \mathrm{ml})$ and reagent III (50 mM 4-chloromercuribenzoic acid, $60 \mathrm{mM} \mathrm{NaOH}, 60 \mathrm{mM}$ EDTA, pH

271 6). After $5 \mathrm{~min}$ at room temperature, the absorbance at $550 \mathrm{~nm}$ was measured for all samples and

272 the average values for biological triplicates of cells with cystatin expression vector were

273 subtracted from the average values for samples of cells with empty vector.

274 Characterization of pMJc01 vector and chicken cystatin reporter in Synechocystis sp. PCC $275 \mathbf{6 8 0 3}$

276 Three biological replicates of Synechocystis sp. PCC 6803 transformed with empty pMJc01 or 277 pMJc01 with two GFP reporter constructs (BBa_I20260_GFPmut3b or L21_RBS*_GFPmut3b) 278 were grown to exponential phase $\left(\mathrm{Abs}_{730}=0.4-0.8\right)$ under standard conditions. Depending on 279 the $\mathrm{Abs}_{730}$ values, 1-2 $\mathrm{ml}$ of the cultures were centrifuged and the pelleted cells resuspended in $280300 \mu 1 \mathrm{TE}$ buffer. Cells were lysed by sonication $(4 \times 1 \mathrm{~min})$ and $52.5 \mu 1$ of the cell lysate was 281 suspended in $2 \mathrm{ml} \mathrm{BG11.} \mathrm{This} \mathrm{resulted} \mathrm{in} \mathrm{an} \mathrm{equivalent} \mathrm{number} \mathrm{of} \mathrm{cells} \mathrm{as} \mathrm{used} \mathrm{for} \mathrm{fluorescence}$ 282 measurements in cultures, with all $S$. sp. PCC 6803 cultures diluted to $\mathrm{Abs}_{730}=0.1$. Fluorescence 283 measurements were performed as described for E. coli.

284 Measurements of GFPmut3b fluorescence at different growth stages were performed using $S$. sp. 285 PCC 6803 transformed with pMJc01_L21_RBS*_GFPmut3b and pMJc01_EV as for E. coli. 286 Cells were diluted to equal cell densities $\left(\mathrm{Abs}_{730}\right)$ at all growth stages.

287 For the papain inhibition assay, cells were prepared as for the GFP fluorescence measurements in cell lysates and $100 \mu \mathrm{l}$ of the lysate was used for the assay, which was performed as described

289 above for $E$. coli, except that the reaction mixture contained $40 \mu 1$ of TE buffer instead of $105 \mu 1$. 


\section{Sequence submission}

291 Nucleotide sequence of pMJc01 with inserted codon-optimized cystatin expression cassette and

292 L21_RBS* regulatory element was deposited in the GenBank database under accession number 293 MN201591.

\section{Results}

\section{Construction of pMJc01, a new shuttle vector for cyanobacteria}

296

297

298

A broad-host range vector pMJc01 was successfully constructed using overlap extension PCR of fragments from plasmids pSB3K3 (Shetty, Endy \& Knight, 2008; cloning site and kanamycin resistance cassette) and RSFmob-I (Katashkina et al., 2007; oriV and rep regions), as shown in Fig. 1. The originally constructed vector also contained the BBa_I20260 BioBrick with GFPmut3b reporter derived from pSB3K3 and was subsequently used to prepare pMJc01 empty vector $(\mathrm{EV})$ and two additional pMJc01 reporter vectors with either GFPmut3b or cystatin reporters under the transcriptional control of L21_RBS*. We prepared this additional regulatory sequence because the BBa_J23101 promoter and BBa_B0032 RBS in the BBa_I20260 BioBrick show low activity in Synechocystis sp. PCC 6803 (Huang \& Lindblad, 2013; Englund, Liang \& Lindberg, 2016). The L21 promoter is based on the TetR-repressible promoter BBa_R0040, which previously showed constitutive expression in $S$. sp. PCC 6803 at approximately 9-fold increase in strength compared to BBa_J23101 (Huang \& Lindblad, 2013). RBS* contains nucleotides complementary to the anti-Shine-Dalgarno sequence of $S$. sp. PCC 6803 and exhibits higher activity in both E. coli and S. sp. PCC 6803 compared to BBa_B0032 (Heidorn et al., 2011; Englund, Liang \& Lindberg, 2016). For comparison with pMJc01, we also prepared 
311 pSB3K3 and pPMQAK1 with the same three reporter cassettes as in pMJc01. For the full list of

312 vectors used, see Table 2.

313 Sequencing of $\mathrm{pMJc} 01$ revealed two same-sense point mutations in the kanamycin resistance

314 gene and a single-base deletion in the His terminator downstream of the cloning site, compared

315 to the sequence of pSB3K3 (retrieved from Registry of Standard Biological Parts). For the

316 mutation detected in the His terminator, we used the RNAfold tool (Hofacker, 2003) to

317 determine the possible effects on the secondary structure and function of the terminator. The

318 deleted nucleotide formed a bulge in the double-stranded stem of the original sequence, which

319 led us to conclude that its absence should not affect the functionality of the region. Comparison

320 of the pMJc01 sequence with the RSFmob-I sequence (GenBank ID: EF467360.1) revealed a

321 two-base substitution in oriV ( $\mathrm{CC}$ to AA, positioned between two iterons) and a single-

322 nucleotide deletion and insertion spaced $6 \mathrm{bp}$ apart in the repC region, causing a short frameshift

323 and substitutions of 3 amino acids (E107D, C108A, H109M). The same two point mutations

324 were observed by Huang and coworkers (Huang et al., 2010) in the sequence of the pPMQAK1

325 plasmid, whose repC region was derived from pAWG1.1 (another RSF1010 derivative plasmid).

326 This indicates that these are most likely not PCR-generated artefacts, but the exact sequence that

327 was originally incorrectly determined for the RSF1010 plasmid.

328

329
pMJc01 is stable in $E$. coli and allows approximately twofold higher reporter protein expression compared to PPMQAK1

To determine the replication capacity of $\mathrm{pMJc} 01$ in $E$. coli, we used the reporter vector pMJc01_L21_RBS*_GFPmut3b (Table 2). We followed GFPmut3b fluorescence at different time points of cell growth in liquid cultures (Fig. 2a), as described in Methods. Fluorescence was 
333 detected at all growth stages with an increase at stationary phase. Over the course of 5 months,

334 we occasionally isolated pMJc01 from E. coli and checked its size and integrity by agarose gel

335 electrophoresis. Reporter expression assays were also repeated several times during this period.

336 We observed no changes in the structure of pMJc01 or in the expression levels driven by this

337 plasmid. On the other hand, pMJc01 showed some structural instability when transferred into the

338 E. coli $\mathrm{DH} 5 \alpha$ strain, a phenomenon that is detailed and discussed in Supplemental Article S1.

339 Reporter protein expression in E. coli was compared between pMJc01, pPMQAK1, and pSB3K3

340 by measuring GFPmut3b fluorescence in the stationary growth phase (Fig. 3). We used two

341 GFPmut3b expression cassettes, which allowed validation of the regulatory sequence L21_RBS*

342 and its comparison with the regulatory sequence J23101_B0032 from BBa_I20260, a reference

343 standard construct in promoter strength measurements (Kelly et al., 2009).

344 Results of relative fluorescence intensities for different plasmids were similar when

345 measurements were performed on bacterial cultures or cell lysates. Also, the results were

346 comparable when GFPmut3b was expressed under the control of L21_RBS* or J23101_B0032

347 regulatory sequences (see Heidorn et al., 2011 for the description of ribosome binding sites and

348 Huang \& Lindblad, 2013 for promoters). Based on the fluorescence intensities, it can be

349 concluded that pMJc01 allows about twofold higher expression compared to pPMQAK1. The

350 expression from pSB3K3 was slightly higher than with pPMQAK1. As judged from fluorescence

351 of cell lysates, the expression ratios pPMQAK1 : pSB3K3 : pMJc01 were $1: 1.3: 2.5$ for

352 reporter constructs with J23101_B0032 regulatory sequence and $1: 1.4: 1.9$ for reporter

353 constructs with L21_RBS* regulatory sequence. Expression ratios based on cell culture

354 fluorescence were $1: 0.5: 1.7$ (for the reporter construct with J23101_B0032 regulatory

355 sequence; the lower value for $\mathrm{pSB} 3 \mathrm{~K} 3$ fluorescence was attributed to measurement artefact due 
356 to greater differences in sample turbidity, see Supplemental Article S1) and $1: 1.1: 2.3$ (for the

357 reporter construct with L21_RBS* regulatory sequence), respectively.

358 Since we expected the higher expression levels with pMJc01 to be explained mainly by the

359 higher plasmid copy numbers in $E$. coli, we decided to roughly determine the absolute average

360 copy numbers of pMJc01 per E. coli cell. For this purpose, we used the quantification of

361 restriction fragments after agarose gel electrophoresis. As a control and for comparison, we also

362 included pSB3K3 in the analysis. Digestion of pMJc01 and pSB3K3 with NotI using two

363 different amounts of isolated plasmids revealed the presence of approximately 18 copies for

$364 \mathrm{pSB} 3 \mathrm{~K} 3$ and 26 copies for $\mathrm{pMJc01}$. In addition, we verified these results with $B s p \mathrm{HI}$ restriction

365 of isolated plasmids, resulting in 17 and 25 copies for pSB3K3 and pMJc01, respectively. We

366 also attempted to include pPMQAK1 in the analysis, but the amount of plasmid isolated from the

367 same number of cells as for the other two plasmids was always too low for quantification.

368 Presumably, this was not due to lower copy number but rather to loss of the plasmid during

369 alkaline lysis (see Discussion).

370 Fluorescence measurements also show that the regulatory sequence L21_RBS* in E. coli is about

371 3-fold stronger than J23101_B0032 with reporter fluorescence ratios in cell lysates of $1: 3.2,1$ :

3723.4 , and $1: 2.4$ when measured with pPMQAK1, pSB3K3, and pMJc01, respectively, and 1 :

$3732.8,1: 6.6$, and $1: 3.7$ in cell cultures. The fluorescence determined with pSB3K3 in cultures

374 differed significantly from the other values, which we attributed to a bias in the fluorescence

375 measurement of samples with different turbidity, as mentioned above and shown in

376 Supplemental Article S1. 
377 Plasmid characterization with cystatin as reporter gave comparable results to GFPmut3b

378 in $E$. coli

379 To evaluate cystatin as a reporter for E. coli and cyanobacteria, a new expression construct with

380 L21_RBS* regulatory sequence was prepared. The complete sequence of the pMJc01 vector

381 with the reporter construct is deposited in GenBank under accession number MN201591. We

382 used this construct to compare the relative copy numbers of pMJc01, pSB3K3, and pPMQAK1

383 in E. coli (Fig. 4a). Two separate experiments were performed, both resulting in ratios

384 comparable to those obtained with GFPmut3b reporter $(1: 1.0: 2.0$ and $1.0: 1.3: 2.5$ in the first

385 and second experiments, respectively, with cystatin as reporter). In addition, we examined the

386 linearity of the response of the papain inhibition assay used (Fig. 4b) and obtained results that

387 were in good agreement with the expected ratio of the signals.

388 Since EV controls were also included in the experiment, we were able to evaluate the inhibitory

389 effect of the chassis on the papain inhibition assay. The $\mathrm{A}_{550}$ values of the samples containing

390 lysates of cells with EV were only slightly lower compared to the control without the addition of

391 cell lysate, therefore we concluded that there are no endogenous inhibitors in E. coli that could

392 interfere with the cystatin reporter assay.

393

394

395

396

397

398

pMJc01 is stable in Synechocystis sp. PCC 6803 and enables strong expression of

\section{GFPmut3b}

To determine the replication ability of pMJc01 in Synechocystis sp. PCC 6803 at different growth stages, we used the reporter vector pMJc01_L21_RBS*_GFPmut3b (Fig. 2b). The fluorescence signal showed quite stable intensity values, which slightly decreased in the exponential growth stage. After three weeks of cultivation, no decrease in fluorescence was 
399 detected, demonstrating stable replication of pMJc01 in Synechocystis sp. PCC 6803 under

400 antibiotic selection pressure. We also examined the amount of expressed GFPmut3b reporter in

401 the stationary growth phase using SDS-PAGE and Coomassie Brilliant Blue staining and saw a

402 distinct band of the appropriate size (26.9 kDa, Fig. 5b), indicating strong reporter expression 403 under the regulatory control of L21_RBS*.

404 In Synechocystis sp. PCC 6803, the regulatory sequences analysed with pMJc01 showed similar 405 reporter fluorescence values when measured in cultures or cell lysates (Fig. 5a). The relative 406 activity of L21_RBS* compared to J23101_B0032 was even higher than in E. coli, with 407 approximately 14-fold higher activity of L21_RBS* (12.4-fold when measured with cell lysates 408 and 15.4-fold when measured with cultures).

409

410

411

412

413

414

415

416

417

418

419

420

\section{Cystatin is a suitable reporter protein for Synechocystis sp. PCC 6803 and showed} approximately 5-fold stronger expression from pMJc01 compared to pPMQAK1

The cystatin expression construct was also analysed in Synechocystis sp. PCC 6803 (Fig. 4c) with vectors pMJc01 and pPMQAK1. We were able to implement the papain inhibition assay for use with Synechocystis sp. PCC 6803 without significant modifications. We only had to optimise the cell lysis step, as this can be more challenging compared to E. coli. Several different approaches were tested (see Supplemental Article S1), but we found that sonication was best for this purpose. Although chemical methods also enabled effective cell lysis or facilitated sonication by decreasing the sonication times required, the chemicals used interfered with the inhibition assay as controls without cell lysate but with lysis buffer added also showed strong papain inhibition. The sonication time was optimised so that after the last sonication interval and centrifugation for $5 \mathrm{~min}$ at $14000 \mathrm{~g}$ there was no green pellet left, indicating complete lysis. We

Peer) reviewing PDF | (2021:06:62918:1:1:NEW 27 Aug 2021) 
421 again used EV controls to determine the background papain inhibition of the chassis and found

422 that it was negligible, although slightly higher than in E. coli. The designed assay was used to

423 compare pMJc01 and pPMQAK1 in Synechocystis sp. PCC 6803. The amount of cystatin

424 detected was approximately 5-fold higher in cells transformed with pMJc01 than in cells

425 transformed with pPMQAK1 (Fig. 4c).

426

\section{Discussion}

428

429

430

431

432

433

434

435

436

437

438

439

440

44

442

Despite some disadvantages that cyanobacteria have compared to established laboratory enterobacteria in genetic engineering, significant progress has been made recently to overcome obstacles and pave the way for cyanobacteria to be considered the "new E. coli" (Ruffing, Jensen \& Strickland, 2016). The main advantages of cyanobacteria, in particular their autotrophic nature and relative ease of genetic manipulation, make these microorganisms very attractive hosts for biotechnology.

We focused on three drawbacks that the majority of currently used episomal vectors and reporters for cyanobacteria have: low biosafety (due to the fact that shuttle vectors contain broad host-range replicators and allow conjugative transfer), low copy number (mostly au pair with the number of genome copies in Synechocystis sp. PCC 6803), and relatively high fluorescent reporter signal backgrounds resulting from cellular components. To address these weaknesses, we designed, constructed, and tested the pMJc01 shuttle plasmid and cystatin as an alternative reporter.

441 The absence of mobilization genes is an essential requirement for improved vector biosafety, as it reduces the likelihood of horizontal gene transfer (Wright, Stan \& Ellis, 2013). The majority of 
443 currently used broad host range replicative plasmids in cyanobacteria (e.g. pPMQAK1) are based

444 on the RSF1010 replicon, which contains mob regions that enable plasmid mobilization in the

445 presence of an auxiliary vector. Our novel pMJc01 plasmid has an RSFmob I-based replicon that

446 lacks the mobilization protein coding sequences as well as the origin of transfer (oriT) and has an

447 undetectable mobilization frequency that is minimally 5 orders of magnitude lower than that of

448 the RSF1010 replicon (Katashkina et al., 2007). Thus, the novel pMJc01 plasmid meets the

449 standard of biosafety (Wright, Stan \& Ellis, 2013). Although this simultaneously means that

450 conjugation is no longer feasible as a means of plasmid transfer to cyanobacteria, we believe this

451 is a small sacrifice, as both electroporation (Chen et al., 2013; Tsujimoto et al., 2015) and natural

452 transformation (Nies et al., 2020) protocols have high efficiencies in many cyanobacterial

453 species.

454 Omission of the RSF1010 mobilization regions further contributes to the superior properties of 455 pMJc01 over RSF1010-based vectors by avoiding loss of the plasmid during isolation by 456 alkaline lysis, a phenomenon that has been described for IncQ plasmids. Kok and coworkers 457 (Kok, Arnberg \& Witholt, 1989) demonstrated that nicking at oriT causes the formation of 458 single-stranded circular DNA, visible on agarose gel after electrophoresis of plasmids isolated by 459 alkaline lysis. A DNA species with high electrophoretic mobility was previously observed for the 460 pPMQAK1 plasmid during our work and also by other researchers (P. Lindblad, personal 461 communications, see Suppl. Fig. 4). Relatively low copy number combined with loss of double462 stranded plasmid DNA during alkaline lysis results in low isolation yield and complicates 463 cloning in E. coli and transfer into cyanobacteria. Since the mob regions are absent in pMJc01, 464 this drawback was not observed during our experimental work. We were always able to isolate 465 decent amounts of pMJc01 from E. coli and had better electroporation efficiency results 
466 compared to pPMQAK1. However, we observed some structural instability of pMJc01 (see

467 Supplemental Article S1), but only when it was propagated under conditions with decreased LacI

468 activity. Based on the observed dependence of structural instability on LacI activity, we

469 speculate that it may be the result of an unknown interaction with $\mathrm{P}_{\text {lacuv }}$, which controls the

470 expression of replication proteins in the RSFmob-I replicon. Unfortunately, we were not able to

471 determine the nucleotide sequence of the elongated plasmid, which could help us to find a

472 mechanistic explanation for this interesting phenomenon. Whether the $\mathrm{P}_{\text {lacUV }}$ is responsible for

473 the observed phenomena could be determined by replacing it with other promoters (an option

474 further discussed in Supplementary Article 1) and observing the stability of the plasmid in

475 different genetic backgrounds. Testing other promoters is also an interesting option in case of

476 unwanted crosstalk in experimental setups using lactose operon-based regulatory systems, as

477 well as for further improvements of $\mathrm{pMJc01}$, since another promoter could increase (or decrease)

478 plasmid copy number.

479 The second goal for the pMJc01 plasmid was to achieve higher copy numbers in both E. coli and 480 Synechocystis sp. PCC 6803. In E. coli, this would facilitate the cloning and construction of 481 synthetic elements to be transferred into cyanobacteria. In $S$. sp. PCC 6803, a higher copy 482 number would allow higher protein expression levels, which are usually difficult to achieve in cyanobacteria but are often desirable. Exceptions include the use of strong regulatory elements

484 such as the "super-strong" $\mathrm{P}_{\mathrm{cbcB}}$ promoter (Zhou et al., 2014) and a combination of the $\mathrm{P}_{\mathrm{psbA}^{*}}$ 485 promoter with two RBS variants (Wang et al., 2018). Although development of stronger 486 promoters has made an important contribution to the cyanobacterial toolbox, plasmids with 487 higher copy numbers might provide more stable and reliable high expression profiles, because 488 for regulatory elements (especially RBS), the specific genetic context can significantly alter their 
489 activity, resulting in different expression levels for different coding sequences (Englund, Liang

$490 \&$ Lindberg, 2016). The use of promoters with lower absolute activities but other desirable

491 properties, such as good inducibility and low leakiness, is also sometimes advantageous. Using

492 pMJc01 in combination with L21_RBS*, we were able to achieve high expression levels of the

493 GFPmut3b reporter in both chassis with visible protein bands in CBB-stained SDS-PAGE gels.

494 Especially in Synechocystis sp. PCC 6803 this is rarely achieved otherwise, neither with

495 episomal nor with chromosomal expression.

496 The novel pMJc01 plasmid has a higher copy number than the RSF1010-based pPMQAK1

497 plasmid in both $E$. coli and $S$. sp. PCC 6803. Although copy numbers were determined by a

498 relatively simple densitometric approach (Projan, Carleton \& Novick, 1983), it is still regularly

499 used (e.g., Valdelvira et al., 2021). Thus, our results provide compelling evidence that pMJc01

500 has an approximately 2-fold higher copy number than pPMQAK1 in E. coli. This was

501 determined using two different reporter systems (fluorescence-based GFPmut3b and cystatin

502 activity) and two different regulatory sequences. These results were also confirmed by plasmid

503 isolation and restriction fragment quantification. The obtained values were in agreement with the

504 results of comparison of RSFmob-I and RSF10101 replicons (Katashkina et al., 2007), where

505 they observed 2-3-fold higher copy number after $16 \mathrm{~h}$ of bacterial growth. Based on the

506 estimated copy number of 10 for RSF1010-based plasmids (Wang et al., 2012), we suggest that

507 pMJc01 has about 20-30 copies per chromosome in E. coli cells in the stationary growth phase,

508 which we also confirmed with our restriction-based quantification. Higher copy number is also

509 reflected in higher plasmid isolation yields. Based on fluorescence measurements at different

510 growth stages, we concluded that $\mathrm{pMJc} 01$ copy number is approximately 2-fold higher in the 
511 stationary growth phase than in the exponential phase, a property previously observed for the

512 RSFmob-I replicon (Katashkina et al., 2007).

513 Using cystatin as a reporter, we compared pMJc01 and pPMQAK1 in Synechocystis sp. PCC

5146803 and showed approximately 5-fold higher reporter expression, which we attributed to the

515 higher copy number of pMJc01. In addition, by GFPmut3b fluorescence measurements, we

516 showed stable replication of pMJc01 in $S$. sp. PCC 6803 over the course of three weeks, with

517 little change in copy number (Fig. 2b). We believe that the high copy number of pMJc01 in $S$. sp.

518 PCC 6803 combined with a relatively strong regulatory sequence contributed to the high

519 absolute levels of the GFPmut3b reporter, as shown by SDS - PAGE of cell lysates.

520 There are many RSF1010-based shuttle backbones that have been tested and used in

521 cyanobacteria, e.g. pPMQAK1 (Huang et al., 2010) and its derivatives (for example pJA2

522 (Anfelt et al., 2013), RSF1010 replicon variants for constructing modular vectors (Taton et al.,

523 2014), pVZ321 (Zinchenko et al., 1999) and its derivatives, such as pSL2680 (Ungerer \&

524 Pakrasi, 2016) and pSL1211 (Ng et al., 2000), and recently tested backbones from the SEVA

525 (Standard European Vector Architecture) repository (Ferreira et al., 2018). These vectors have

526 been used for a range of studies, from characterization of synthetic biology genetic elements

527 (Huang \& Lindblad, 2013; Camsund, Heidorn \& Lindblad, 2014; Badary et al., 2015; Englund,

528 Liang \& Lindberg, 2016) to protein overexpression (Anfelt et al., 2013; Ng et al., 2000),

529 including genome-modifying enzymes (Ungerer \& Pakrasi, 2016). Despite the widespread use of

530 RSF1010-based vectors, RSFmob-I-based plasmids, such as pMJc01, can be considered an ideal

531 alternative for a variety of applications due to their stability during plasmid isolation, higher

532 copy number in both E. coli and cyanobacteria, improved biosafety, and easier manipulation.

533 Although some backbones devised from the native Synechocystis sp. PCC 6803 plasmids (e.g. 
534 (Armshaw et al., 2015; Jin et al., 2018)) also show higher copy numbers compared to RSF1010-

535 based plasmids, they still lack the broad host range, another advantage of RSFmob-I-based

536 plasmids.

537 Protein reporters are indispensable tools for the quantitative characterization of regulatory

538 elements involved in the assembly of complex genetic circuits (Decoene et al., 2018). To this

539 end, reporters should be functional in the chosen microbial strain and have a broad dynamic

540 range covering common expression levels. Additionally, it is desirable that they allow for both

541 high-throughput and single-cell analyses (Martin, Che \& Endy, 2009). In cyanobacteria,

542 fluorescent proteins are most commonly used as reporters, but they can have notable drawbacks

543 because photosynthetic pigments in cyanobacteria can potentially interfere with the measured

544 fluorescence signal by competing for the absorption of excitation light, with the re-absorption of

545 emitted fluorescence, and by emitting intrinsic fluorescence, that affects the excitation or

546 emission of the recombinant fluorescent proteins. Therefore, the selection of fluorescent

547 reporters with spectral properties that cause minimal interference with the cellular background is

548 critical (Ruffing, Jensen \& Strickland, 2016). The use of non-fluorescent reporters for

549 cyanobacteria could therefore be beneficial and independent of the species or strain used.

550 Moreover, it could also allow orthogonality when using light-inducible regulatory systems,

551 which have already been successfully implemented in cyanobacteria (Badary et al., 2015) and

552 whose activity could change with exposure to the excitation light used in reporter measurements.

553 To this end, we tested cystatin, a $13 \mathrm{kDa}$ protein inhibitor of cysteine proteases that has been

554 used previously as a qualitative reporter (Škrlj, Erčulj \& Dolinar, 2009) but never as a

555 quantitative reporter to characterise regulatory elements in vectors. Among several cystatins, we

556 chose hen egg white cystatin, a stable and highly efficient inhibitor of papain and related 
557 proteases (Ki values in the pM range) (Machleidt et al., 1989). Cystatin expression was

558 successful in both E. coli and Synechocystis sp. PCC 6803, and cystatin expression-based

559 plasmid characterization in E. coli yielded comparable results to those obtained with GFPmut3b,

560 demonstrating the potential of cystatin for quantitative characterization of regulatory genetic

561 elements. The latter is further supported by the linear response of activity against the amount of

562 cell lysate and thus against cystatin concentration. In Synechocystis sp. PCC 6803, we could

563 directly compare cystatin production from pPMQAK1 and pMJc01 plasmids and detected

564 significantly higher cystatin expression with pMJc01.

565 Although the expressed levels of cystatin appeared to be lower than those of GFPmut3b the

566 latter was detectable by SDS -PAGE and Coomassie Brilliant Blue staining in both organisms,

567 whereas cystatin was not), it still allowed quantification by papain inhibition assay. This

568 correlates with a known advantage of reporter systems based on enzymatic activity over

569 fluorescent proteins - enzymes allow signal amplification, whereas with fluorescent probes one

570 molecule provides one fluorophore that will photobleach with time, making detection of weakly

571 expressed proteins difficult (Martin, Che \& Endy, 2009). The quantification range of cystatin

572 could be further increased by using fluorescent papain substrates (Škrlj, Erčulj \& Dolinar, 2009).

573 Cystatin lacks the main advantage of fluorescent reporters, namely in vivo real-time

574 measurements. Quantification of cystatin requires cell lysis, which can be complicated by the

575 presence of strong cell wall structures in some microorganisms, such as cyanobacteria. We had

576 to test and adapt several protocols to achieve a satisfactory lysis compatible with the papain

577 inhibition assay. On the other hand, we also found some biases in in vivo measurements with the

578 GFPmut3b fluorescent reporter. Namely, we observed a strong inner filter effect when measuring

579 fluorescence of E. coli cultures (see Supplemental Article S1), which causes erroneous 
580 normalization because the calculation of fluorescence per cell (the usual way of processing

581 fluorescence measurements) can differ greatly when measuring at different optical densities.

582 Although this can be solved by diluting all measured samples to the same optical density (as was

583 done in our experiments), this is not usually the practice as it detracts from the simplicity of

584 parallel in-plate measurements. Moreover, it is not practical for continuous in vivo

585 measurements. In light of the above, we believe that although data acquisition is simpler with

586 fluorescent proteins, making them valuable for rapid high-throughput screening, measurement

587 bias, either due to the inner filter effect or other interactions with endogenous pigments, may be

588 unacceptable for more accurate and absolute characterizations of biological parts. We believe

589 that the use of an alternative reporter with a different type of signal generation should be used for

590 verification of fluorescence measurements of biological parts of interest, and we propose cystatin

591 as a promising reporter.

592 Another strong motive for using different reporters to characterize biological parts, especially

593 regulatory sequences, is the strong sequence-specific influence of the downstream gene on their

594 activity. For both promoters and RBS, it has been consistently shown that genetic context

595 strongly influences expression levels (Englund, Liang \& Lindberg, 2016). The use of different

596 reporters has already been adopted for a detailed analysis of RBS in Synechocystis sp. PCC 6803

597 (Thiel et al., 2018).

\section{Conclusions}

599 The evidence presented shows that the pMJc01 plasmid based on the RSFmob-I replicon has

600 higher copy number in E. coli and Synechocystis sp. PCC 6803 and better biosafety and stability

601 compared to the common RSF1010-based broad host-range plasmid pPMQAK1. It can be used 
602 for easy cloning and biological part construction in E. coli as well as for protein expression in 603 Synechocystis sp. PCC 6803. In both chassis, it should also prove useful for the characterization 604 of genetic elements.

605 We also introduce a new non-fluorogenic reporter, cystatin, which is active and useful in both $E$. 606 coli and Synechocystis sp. PCC 6803. It provides an alternative to fluorescent reporters in 607 experimental setups where they are not applicable, as well as a useful tool for verifying the 608 results of fluorescence-based measurements.

\section{Acknowledgements}

610 We would like to thank Dr. Joanna Katashkina from Ajinomoto-Genetika Research Institute,

611 Moscow, for her help in obtaining the RSFmob-I plasmid from the Russian National Collection 612 of Industrial Microorganisms.

\section{References}

615 Anfelt J, Hallström B, Nielsen J, Uhlén M, Hudson EP. 2013. Using transcriptomics to improve 616 butanol tolerance of Synechocystis sp. strain PCC 6803. Applied and Environmental Microbiology 79(23):7419-7427. DOI: 10.1128/AEM.02694-13.

Armshaw P, Carey D, Sheahan C, Pembroke JT. 2015. Utilising the native plasmid, pCA2.4, 619 from the cyanobacterium Synechocystis sp. strain PCC6 803 as a cloning site for enhanced 620 product production. Biotechnology for Biofuels 8:201. DOI: 10.1186/s13068-015-0385-x

621 Badary A, Abe K, Ferri S, Kojima K, Sode K. 2015. The development and characterization of an 
622

623

624

625

626

627

628

629

630

631

632

633

634

635

636

637

638

639

640

641

642

exogenous green-light-regulated gene expression system in marine cyanobacteria. Marine Biotechnology 17(3):245-251. DOI: 10.1007/S10126-015-9616-1.

Badary A, Sode K. 2020. Marine Cyanobacteria. In: Se-Kwon K, ed. Encyclopedia of Marine Biotechnology. Hoboken: Wiley-Blackwell, 2127-2146.

Camsund D, Heidorn T, Lindblad P. 2014. Design and analysis of LacI-repressed promoters and DNA-looping in a cyanobacterium. Journal of Biological Engineering 8:4. DOI: 10.1186/1754-1611-8-4.

Chen H, Hanzhi L, Peng J, Fuchao L, Song Q. 2013. Genetic transformation of marine cyanobacterium Synechococcus sp. CC9311 (Cyanophyceae) by electroporation. Chinese Journal of Oceanology and Limnology 31(2):416-420. DOI: 10.1007/S00343-013-2164-5.

Chen Y, Taton A, Go M, London RE, Pieper LM, Golden SS, Golden JW. 2016. Self-replicating shuttle vectors based on pANS, a small endogenous plasmid of the unicellular cyanobacterium Synechococcus elongatus PCC 7942. Microbiology 162(12):2029-2041. DOI: $10.1099 /$ mic. 0.000377.

Cormack BP, Valdivia RH, Falkow S. 1996. FACS-optimized mutants of the green fluorescent protein (GFP). Gene 173(1):33-38. DOI: 10.1016/0378-1119(95)00685-0.

Decoene T, De Paepe B, Maertens J, Coussement P, Peters G, De Maeseneire SL, De Mey M. 2018. Standardization in synthetic biology: an engineering discipline coming of age. Critical Reviews in Biotechnology 38(5):647-656. DOI: 10.1080/07388551.2017.1380600.

Englund E, Liang F, Lindberg P. 2016. Evaluation of promoters and ribosome binding sites for biotechnological applications in the unicellular cyanobacterium Synechocystis sp. PCC 
6803. Scientific Reports 6:36640. DOI: 10.1038/srep36640.

644 Ferreira EA, PAcheco CC, Pinto F, Pereira J, Lamosa P, Oliveira P, Kirov B, Jaramillo A, 645 Tamagnini P. 2018. Expanding the toolbox for Synechocystis sp. PCC 6803: validation of 646 replicative vectors and characterization of a novel set of promoters. Synthetic Biology 647 3(1):ysy014 DOI: 10.1093/synbio/ysy014.

648 Fossum K, Whitaker JR. 1968. Ficin and papain inhibitor from chicken egg white. Archives of 649 Biochemistry and Biophysics 125(1):367-375. DOI: 10.1016/0003-9861(68)90672-3.

650 Frey J, Bagdasarian M. 1989. The molecular biology of IncQ plasmids. In: Thomas CM, ed. 651 Promiscuous Plasmids of Gram-negative Bacteria. London: Academic Press, 79-94.

652 Hashiro S, Yasueda H. 2018. Plasmid copy number mutation in repA gene encoding RepA 653 replication initiator of cryptic plasmid pHM1519 in Corynebacterium glutamicum. 654 Bioscience, Biotechnology and Biochemistry 82(12):2212-2224. DOI: $655 \quad 10.1080 / 09168451.2018 .1508986$.

656 Heidorn T, Camsund D, Huang H-H, Lindberg P, Oliveira P, Stensjö K, Lindblad P. 2011.

657 Synthetic biology in yyanobacteria: engineering and analyzing novel functions. Methods in 658 Enzymology 497:539-579. DOI: 10.1016/B978-0-12-385075-1.00024-X.

659 Hitchcock A, Hunter CN, Canniffe DP. 2020. Progress and challenges in engineering 660 cyanobacteria as chassis for light-driven biotechnology. Microbial Biotechnology 661 13(2):363-367. DOI: 10.1111/1751-7915.13526.

662 Hofacker IL. 2003. Vienna RNA secondary structure server. Nucleic Acids Research 663 31(13):3429-3431. DOI: 10.1093/nar/gkg599. 
664 Huang, H-H, Camsund D, Lindblad P, Heidorn T. 2010. Design and characterization of 665 molecular tools for a synthetic biology approach towards developing cyanobacterial 666 biotechnology. Nucleic Acids Research 38(8):2577-2593. DOI: 10.1093/nar/gkq164.

667 Huang H-H, Lindblad P. 2013. Wide-dynamic-range promoters engineered for cyanobacteria. 668 Journal of biological engineering 7(1):10. DOI: 10.1186/1754-1611-7-10.

669

670

671

672

673

674

675

676

677

678

679

680

681

682

683

684

685

Hudson EP. 2021. Synthetic Biology in Cyanobacteria and Applications for Biotechnology. In: Nielsen J, Lee S, Stephanopoulos G, Hudson P, eds. Cyanobacteria Biotechnology. Weinheim: WILEY-VCH, 123-170.

Jahn M, Vorpahl C, Hübschmann T, Harms H, Müller S. 2016. Copy number variability of expression plasmids determined by cell sorting and droplet digital PCR. Microbial Cell Factories 15(1):211. DOI: 10.1186/s12934-016-0610-8.

Jin H, Wang Y, Idoine A, Bhaya D. 2018. Construction of a shuttle vectoru an endogenous plasmid from the cyanobacterium Synechocystis sp. PCC 6803. Frontiers in Microbiology 9:1662. DOI: 10.3389/fmicb.2018.01662.

Katashkina JI, Kuvaeva T, Andreeva IG, Skorokhodova AY, Biryukova IV, Tokmakova IL, Golubeva LI, Mashko SV. 2007. Construction of stably maintained non-mobilizable derivatives of RSF1010 lacking all known elements essential for mobilization. BMC biotechnology 7:80. DOI: 10.1186/1472-6750-7-80.

Kelly JR, Rubin AJ, Davis JH, Ajo-Franklin CM, Cumbers J, Czar MJ, de Mora K, Glieberman AL, Monie DD, Endy D. 2009. "Measuring the Activity of BioBrick Promoters Using an in Vivo Reference Standard.” Journal of Biological Engineering 3:1-13. DOI: 10.1186/17541611-3-4. 
686 Khan AZ, Bilal M, Mehmood S, Sharma A, Iqbal HMN. 2019. State-of-the-art genetic

687 modalities to engineer cyanobacteria for sustainable biosynthesis of biofuel and fine688 chemicals to meet bio-economy challenges. Life 9(3):1-22 DOI: 10.3390/life9030054.

689 Klemenčič M, Nielsen AZ, Sakuragi Y, Frigaard N-U, Čelešnik H, Jenden PE, Dolinar M. 2017. 690 Synthetic Biology of Cyanobacteria for Production of Biofuels and High-Value Products.

691 In: Gonzalez-Fernandez C, Muñoz R, ed. Microalgae-Based Biofuels and Bioproducts.

692 Sawston: Woodhead Publishing, 305-325.

693

694

695

696

697

698

699

700

701

702

703

704

705

706

707

Knight T. 2003. Idempotent Vector Design for Standard Assembly of Biobricks. Available at: https://dspace.mit.edu/handle/1721.1/21168 (accessed 5 August 2020)

Kok M, Arnberg AC, Witholt B. 1989. Single-stranded circular DNA generated from broad host range plasmid R1162 and its derivatives. Plasmid 21(3):238-241. DOI: 10.1016/0147619X(89)90047-4.

Kopitar M, Brzin J, Zvonar T, Ločnikar P, Kregar I, Turk V. 1978. Inhibition studies of an intracellular inhibitor on thiol proteinases. FEBS 91(2):355-359. DOI: 10.1016/00145793(78)81209-5.

Lindblad P, Fuente D, Borbe F, Cicchi B, Conejero JA, Couto N, Čelešnik H, Diano MM, Dolinar M, Esposito S, Evans C, Ferreira EA, Keller J, Khanna N, Kind G, Landels A, Lemus L, Noirel J, Ocklenburg S, Oliveira P, Pacheco CC, Parker JL, Pereira J, Pham TK, Pinto F, Rexroth S, Rögner M, Schmitz H-J, Benavides AMS, Siurana M, Tamagnini P, Touloupakis E, Torzillo G, Urchueguía JF, Wegelius A, Wiegand K, Wright PC, Wutschel M, Wünschiers R. 2019. CyanoFactory, a European consortium to develop technologies needed to advance cyanobacteria as chassis for production of chemicals and fuels. Algal 
Research 41:101510. DOI: 10.1016/j.algal.2019.101510.

709

710

711

712

713

714

715

716

717

718

719

720

721

722

723

724

725

726

727

728

Liu D, Liberton M, Hendry JI, Aminian-Dehkordi J, Maranas CD, Pakrasi HB. 2021.

Engineering biology approaches for food and nutrient production by cyanobacteria. Current Opinion in Biotechnology 67:1-6. DOI: 10.1016/j.copbio.2020.09.011.

Ma AT, Schmidt CM, Golden JW. 2014. Regulation of gene expression in diverse cyanobacterial species by using theophylline-responsive riboswitches. Applied and Environmental Microbiology 80(21):6704-6713. DOI: 10.1128/AEM.01697-14.

Machleidt W, Thiele U, Laber B, Assfalg-Machleidt I, Esterl A, Wiegand G, Kos J, Turk V, Bode W. 1989. Mechanism of inhibition of papain by chicken egg white cystatin. Inhibition constants of $\mathrm{N}$-terminally truncated forms and cyanogen bromide fragments of the inhibitor. FEBS Letters 243(2):234-238. DOI: 10.1016/0014-5793(89)80135-8.

Martin L, Che A, Endy D. 2009. Gemini, a bifunctional enzymatic and fluorescent reporter of gene expression. PLoS ONE 4(11):e7569. DOI: 10.1371/journal.pone.0007569.

Mermet-Bouvier P, Cassier-Chauvat C, Marraccini P, Chauvat F. 1993. Transfer and replication of RSF1010-derived plasmids in several cyanobacteria of the genera Synechocystis and Synechococcus. Current Microbiology 27(6):323-327. DOI: 10.1007/BF01568955.

Meyer R. 2009. Replication and conjugative mobilization of broad host-range IncQ plasmids. Plasmid 62(2):57-70. DOI: 10.1016/j.plasmid.2009.05.001.

Ng I-S, Keskin BB, Tan S-I. 2020. A critical review of genome editing and synthetic biology applications in metabolic engineering of microalgae and cyanobacteria. Biotechnology Journal 15(8):1900228. DOI: 10.1002/biot.201900228. 
729 Ng W-O, Zentella R, Wang Y, Taylor J-SA, Pakrasi HB. 2000. PhrA, the major

730 photoreactivating factor in the cyanobacterium Synechocystis sp. strain PCC 6803 codes for

731 a cyclobutane-pyrimidine-dimer-specific DNA photolyase. Archives of Microbiology

$732 \quad$ 173:412-417. DOI: 10.1007/s002030000164.

733 Nies F, Mielke M, Pochert J, Lamparter T. 2020. Natural transformation of the filamentous

$734 \quad$ cyanobacterium Phormidium lacuna. PLoS ONE 15(6):e0234440. DOI:

735 10.1371/journal.pone.0234440.

736

737

738

739

740

741

742

743

744

745

746

747

748

749

Pinto F, Pacheco CC, Oliveira P, Montagud A, Landels A, Couto N, Wright PC, Urchueguía JF, Tamagnini P. 2015. Improving a synechocystis-based photoautotrophic chassis through systematic genome mapping and validation of neutral sites. DNA Research 22(6):425-437. DOI: $10.1093 /$ dnares/dsv024.

Projan SJ, Carleton S, Novick RP. 1983. Determination of plasmid copy number by fluorescence densitometry. Plasmid 9(2):182-190. DOI: 10.1016/0147-619X(83)90019-7.

Ruffing AM., Jensen TJ, Strickland LM. 2016. Genetic tools for advancement of Synechococcus sp. PCC 7002 as a cyanobacterial chassis. Microbial Cell Factories 15(1):190. DOI: 10.1186/s12934-016-0584-6.

Santos-Merino M, Sing AK, Ducat DC. 2019. New applications of synthetic biology tools for cyanobacterial metabolic engineering. Frontiers in Bioengineering and Biotechnology 7:33. DOI: $10.3389 /$ fbioe. 2019.00033 .

Shetty RP, Endy D, Knight TF. 2008. Engineering BioBrick vectors from BioBrick parts. Journal of Biological Engineering 2:5. DOI: 10.1186/1754-1611-2-5. 
750 Škrlj N, Erčulj E, Dolinar M. 2009. A versatile bacterial expression vector based on the synthetic 751 biology plasmid pSB1." Protein Expression and Purification 64(2):198-204.

752 Sproles AE, Fields FJ, Smalley TN, Le CH, Badary A, Mayfield SP. 2021. Recent advancements 753 in the genetic engineering of microalgae. Algal Research 53:102158. DOI:

754

Stanier RY, Kunisawa R, Mandel M, Cohen-Bazire G. 1971. Purification and properties of 10.1016/j.algal.2020.102158

Taniuchi Y, Murakami A, Ohki K. 2008. Whole-cell immunocytochemical detection of nitrogenase in cyanobacteria: improved protocol for highly fluorescent cells. Aquatic

Taton A, Unglaub F, Wright NE, Zeng WY, Paz-Yepes J, Brahamsha B, Palenik B, Peterson TC, Haerizadeh F, Golden SS, Golden JW. 2014. Broad-host-range vector system for synthetic Microbial Ecology 51(3):237-247. DOI: 10.3354/ame01197.

765

766

767

768

769

770

771 unicellular blue-green algae (order Chroococcales). Bacteriological reviews 35(2):171-205. DOI: 10.1128/br.35.2.171-205.1971. biology and biotechnology in cyanobacteria. Nucleic Acids Research 42(17):e136. DOI: 10.1093/nar/gku673.

Thiel K, Mulaku E, Dandapani H, Nagy C, Aro E-M, Kallio P. 2018. Translation efficiency of heterologous proteins is significantly affected by the genetic context of RBS sequences in engineered cyanobacterium Synechocystis sp. PCC 6803. Microbial Cell Factories 17:34. DOI: $10.1186 / \mathrm{s} 12934-018-0882-2$.

Thompson MG, Sedaghatian N, Barajas JF, Wehrs M, Bailey CB, Kaplan N, Hillson NJ, Mukhopadhyay A, Keasling JD. 2018. Isolation and characterization of novel mutations in the pSC101 origin that increase copy number. Scientific Reports 8(1):1590. DOI: 
773

774

775

776

777

778

779

780

781

782

783

784

785

786

787

788

789

790

791

792
Tsujimoto R, Kotani H, Nonaka A, Miyahara Y, Hiraide Y, Fujita Y. 2015. Transformation of the cyanobacterium Leptolyngbya boryana by electroporation. Bio-protocol 5(24):e1690. DOI: 10.21769/BioProtoc.1690.

Turk V, Stoka V, Turk D. 2008. Cystatins: biochemical and structural properties, and medical relevance. Frontiers in Bioscience 13(14):5406-5420. DOI: 10.2741/3089.

Ungerer J, Pakrasi HB. 2016. Cpf1 is a versatile tool for CRISPR genome editing across diverse species of cyanobacteria. Scientific Reports 6:39681. DOI: 10.1038/srep39681.

Valdelvira R, Bordanaba-Ruiseco L, Martín-Huestmendía C, Ruiz-Masó JA, del Solar G. 2021. Acidic $\mathrm{pH}$ decreases the endonuclease activity of initiator RepB and increases the stability of the covalent RepB-DNA intermediate while has only a limited effect on the replication of plasmid pMV158 in Lactococcus lactis. Frontiers in Molecular Biosciences 8:634461. DOI: 10.3389/fmolb.2021.634461.

Vavitsas K, Crozet P, Vinde MH, Davies F, Lemaire SD, Vickers CE. 2019. The synthetic biology toolkit for photosynthetic microorganisms. Plant Physiology 181(1):14-27. DOI: $10.1104 /$ pp. 19.00345

Vioque A. 2007. Transformation of cyanobacteria. Advances in Experimental Medicine and Biology 616:12-22. DOI: 10.1007/978-0-387-75532-8_2.

Wang B, Eckert C, Maness P-C, Yu J. 2018. A genetic toolbox for modulating the expression of heterologous genes in the cyanobacterium Synechocystis sp. PCC 6803. ACS Synthetic Biology 7(1):276-286. DOI: 10.1021/acssynbio.7b00297.

Peer] reviewing PDF | (2021:06:62918:1:1:NEW 27 Aug 2021) 
793 Wang B, Wang J, Zhang W, Meldrum DR. 2012. Application of synthetic biology in

$794 \quad$ cyanobacteria and algae. Frontiers in Microbiology 3:344. DOI:

795 10.3389/fmicb.2012.00344.

796

797

798

799

800

801

802

803

804

805

806

807

808

809

810

811

812

Wang F, Gao Y, Yang G. 2020. Recent advances in synthetic biology of cyanobacteria for improved chemicals production. Bioengineered 11(1):1208-1220. DOI: $10.1080 / 21655979.2020 .1837458$

Wright O, Stan G-B, Ellis T. 2013. Building-in biosafety for synthetic biology. Microbiology 159:1221-1235. DOI: 10.1099/mic.0.066308-0.

Zang X, Liu B, Liu S, Arunakumara KKIU, Zhang X. 2007. Optimum conditions for transformation of Synechocystis sp. PCC 6803. The Journal of Microbiology 45(3):241245.

Zerulla K, Ludt K, Soppa J. 2016. The ploidy level of Synechocystis sp. PCC 6803 is highly variable and is influenced by growth phase and by chemical and physical external parameters. Microbiology 162(5):730-739. DOI: 10.1099/mic.0.000264.

Zhang W, Song X. 2018. Synthetic Biology of Cyanobacteria. Singapore: Springer.

Zhou J, Zhang H, Meng H, Zhu Y, Bao G, Zhang Y, Li Y, Ma Y. 2014. Discovery of a superstrong promoter enables efficient production of heterologous proteins in cyanobacteria. Scientific Reports 4(1):4500. DOI: 10.1038/srep04500.

Zinchenko V, Piven I, Melnik V, Shestakov S. 1999. Vectors for the complementation analysis of cyanobacterial mutants. Russian Journal of Genetics 35(3):228-232. 


\section{Figure 1}

Plasmid maps of the broad host range pMJc01 vector and its vectors of origin, RSFmob-I and $\mathrm{pSB} 3 \mathrm{~K} 3$

The new pMJc01 vector was obtained after splicing by PCR of two fragments amplified from plasmids RSFmob-I and pSB3K3 (marked with grey circular sectors). From RSFmob-I, we amplified the replicator with oriV and replicator protein genes (encoding RepB, OrfE, OrfF, RepA, RepC) under the transcriptional control of the lacUV5 promoter and the lac operator. The pSB3K3-derived regions included the kanamycin resistance casette and the BioBrick cloning site with prefix, suffix, and two terminators $(T)$ at both ends. Our original construct also contained the BBa_I20260 BioBrick. 

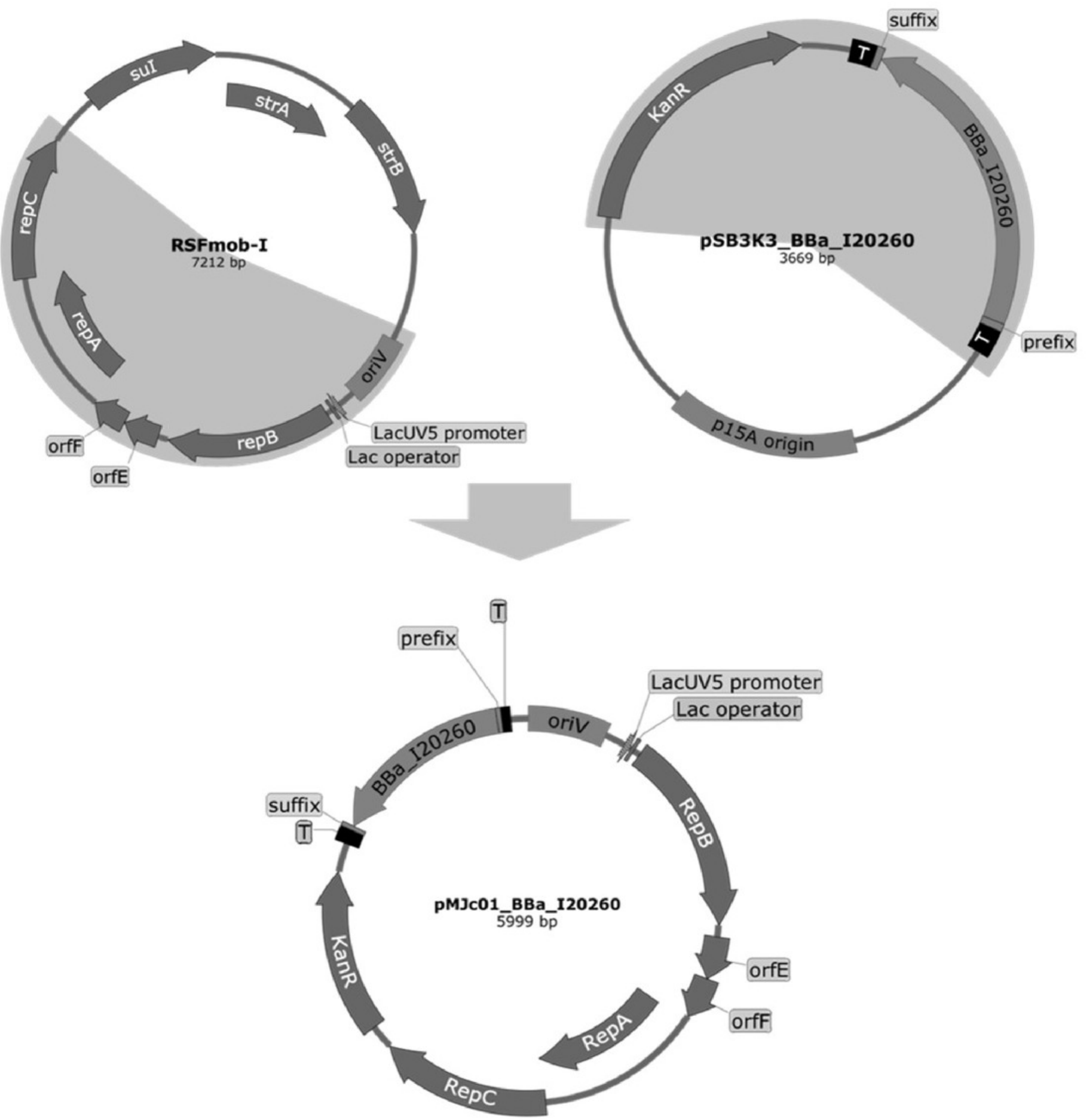


\section{Figure 2}

GFPmut3b fluorescence in cultures of E. coli and Synechocystis sp. PCC 6803 at different growth stages

Fluorescence of cultures transformed with empty pMJc01 vector (EV) or pMJC01_L21_RBS*_GFPmut3b (GFP) was measured at different time points. Cultures were diluted to Abs600 (for E. coli) or Abs730 (for S. sp. PCC 6803) of 0.1, except for the first two measurements where absorbance values were lower and the measured fluorescence values were extrapolated to an absorbance of 0.1. Biological triplicates were used (except for E. coli transformed with empty $\mathrm{pMJc01)}$ and fluorescence values are reported as the difference between the average values for samples with EV and samples with GFPmut3b expression device. Where applicable, standard deviation values are given as error bars. (a) Growth curves of $E$. coli with (GFP, squares) or without (EV, diamonds) GFPmut3b expression device and fluorescence intensity (triangles). The time scale on the x-axis is exponential. (b) Growth curves of Synechocystis sp. PCC 6803 with (GFP, squares) or without (EV, diamonds) GFPmut3b expression device and fluorescence intensity (triangles).
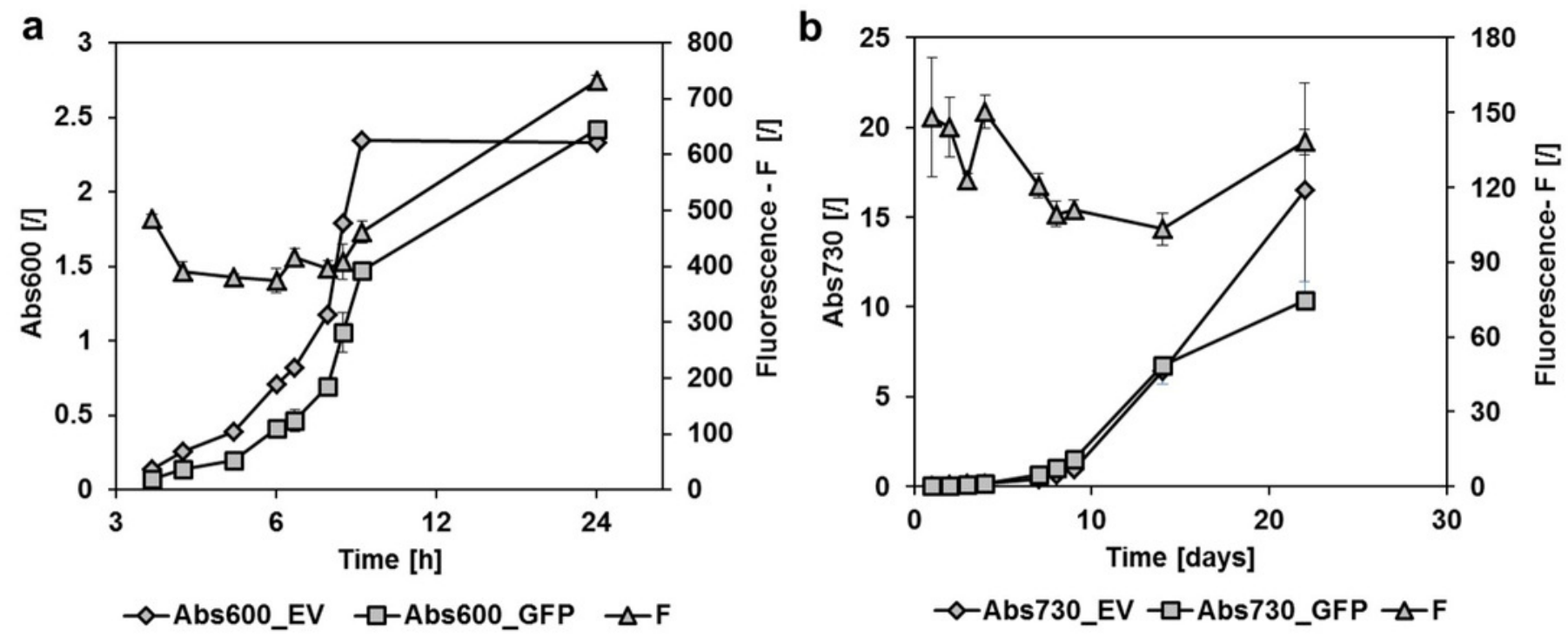


\section{Figure 3}

Relative GFPmut3b reporter expression from different plasmid backbones

GFPmut3b fluorescence intensity in $E$. coli transformed with pMJc01, pSB3K3, or pPMQA1 containing different regulatory sequences in the reporter expression device. Shown are differences between mean values for triplicates of cells with expressed GFPmut3b and cells with empty vector \pm standard deviation. (a) GFPmut3b fluorescence intensity in cell cultures of E. coli. (b) GFPmut3b fluorescence intensity in cell lysates of E. coli.

a

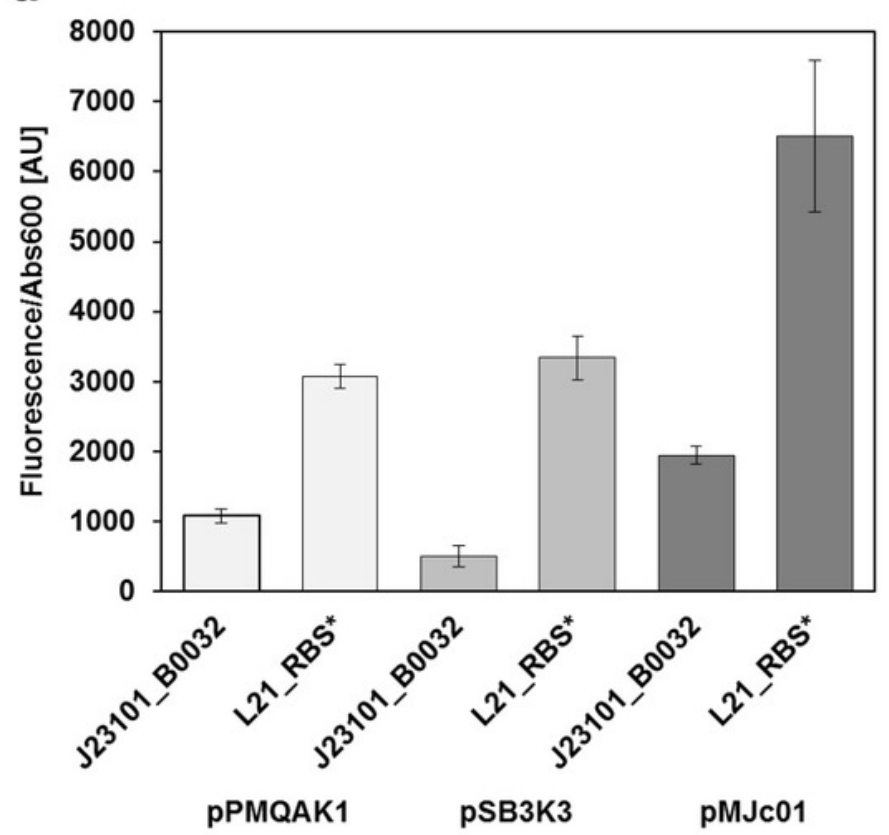

b

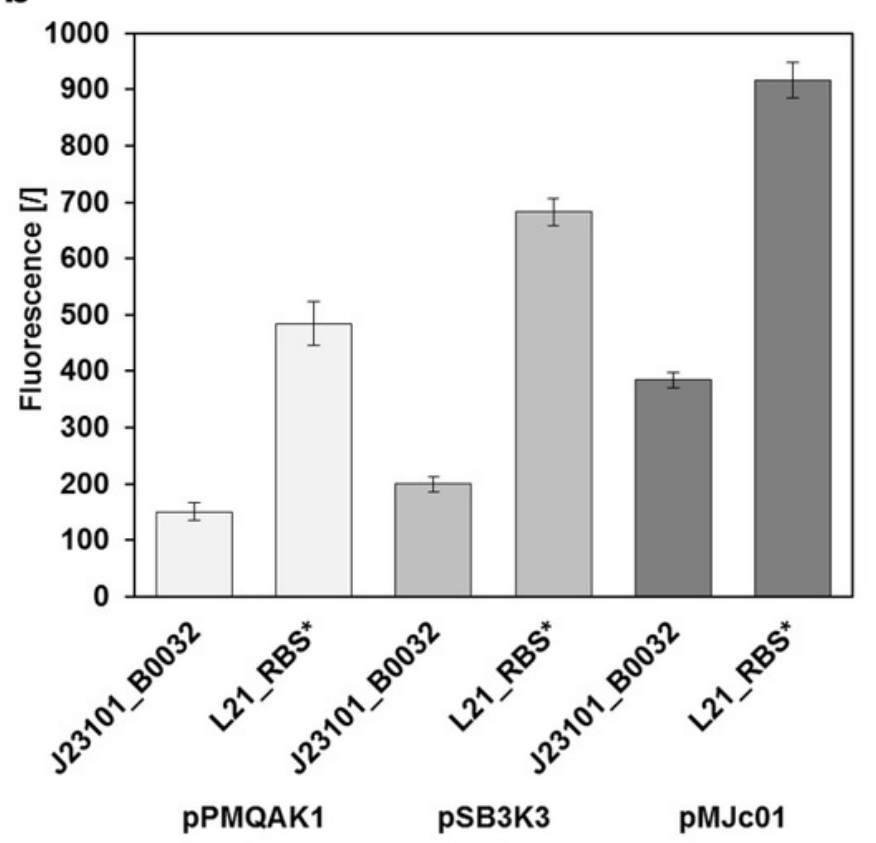




\section{Figure 4}

Analysis of chicken cystatin as a reporter in E. coli and Synechocystis sp. PCC 6803

Expression of chicken cystatin was determined with papain inhibition assay - high $\Delta \mathrm{Abs} 550$ values indicate stronger inhibition and therefore higher quantities of cystatin in the tested lysates. Error bars represent \pm standard deviation. (a) Relative plasmid copy number evaluation in E. coli with cystatin reporter. Light and dark gray bars represent two replications of the same experiment. The absolute values for both experiments were normalized ( 1 being the measurement with pMJc01) as the amount of cell material differed between repetitions. (b) Linearity of response for papain inhibition assay. 10, 15 or $30 \mu$ of cell lysate (E. coli transformed with pMJc01 and cystatin expression device) were used. Samples were measured in duplicates. (c) Evaluation of cystatin reporter expression from plasmids pMJc01 and pPMQAK1 in Synechocystis sp. PCC 6803.
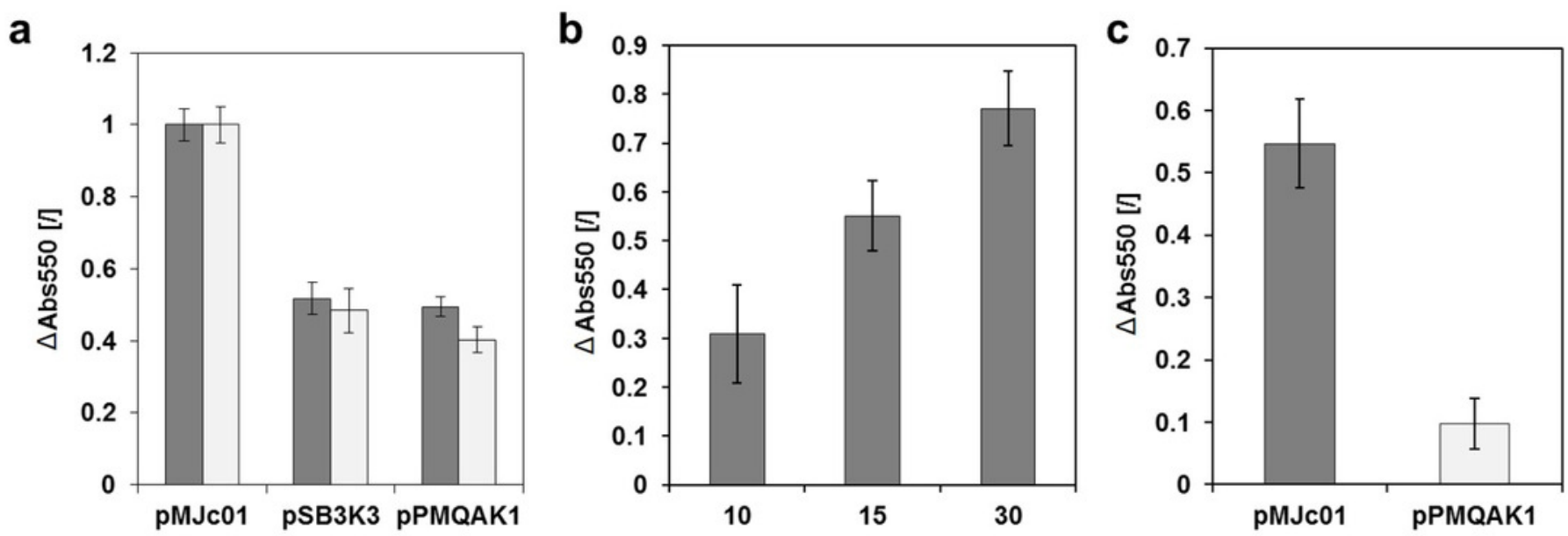


\section{Figure 5}

Expression of GFPmut3b in Synechocystis sp. PCC 6803 transformed with pMJc01 vector

(a )GFPmut3b fluorescence of cell lysates and cultures of cyanobacteria Synechocystis sp.

PCC 6803, transformed with pMJC01 vector with GFPmut3b expression under control of

L21_RBS* or J23101_B0032. Error bars represent \pm standard deviation. (b) SDS-PAGE of

Synechocystis sp. PCC 6803 cell lysates; EV - cells transformed with empty pMJc01, C - cells transformed with pMJC01_L21_RBS*_cystatin6803, GFPw - cells transformed with pMJC01_BBa_I20260, GFPs - cells transformed with pMJC01_L21_RBS*_GFPmut3b. The protein band corresponding to GFPmut3b is marked with an arrow.

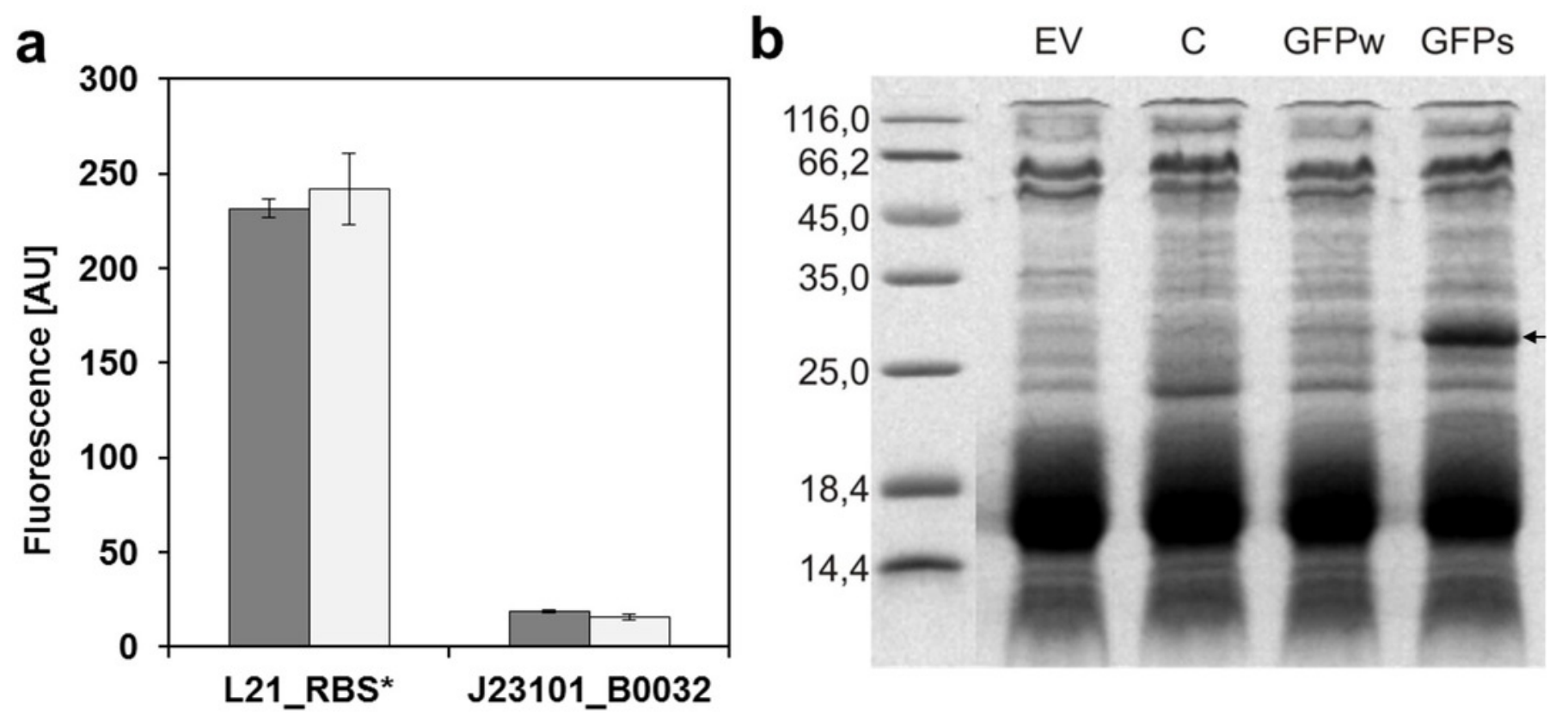

$\square$ cell lysates $\square$ cell cultures 


\section{Table $\mathbf{1}$ (on next page)}

List of primers used in this study

All primers (except VF2 - BBa_G00100 and VR - BBa_G00101, see Registry of Standard Biological Parts, http://parts.igem.org/Main_Page) were designed as part of this study. Underlined are $18 \mathrm{nt}$ long overlapping sequences that were used for the overhang assembly of pMJc01. 


\begin{tabular}{cl}
\hline Primer name & \\
\hline RSFmobF & AACTGTCACGAACCCCTGCAATAACTGT \\
\hline RSFmobR & TTTGTTGAATGGGTCAGCCTGCCGCCTT \\
\hline pSB3K3F & GCTGACCCATTCAACAAAGCCACGTTGTGT \\
& \\
\hline pSB3K3R & TTCCATGGTGCCACCTGACGTCTAAGA \\
& \\
\hline \multirow{2}{*}{ L21_RBS*_F } & GCTCTAGAGGGGCCCTCCCTATCAGTGATAGAGATTGACATCCCTATC \\
& AGTGATAGATATAATGGGAGCTACTAGAGTAGTGGAGGTTACTAGTCG \\
& \\
\hline & CGACTAGTAACCTCCACTACTCTAGTAGCTCCCATTATATCTATCACT \\
&
\end{tabular}

GFPmut3bF GCTCTAGATGCGTAAAGGAGAAGA

VR ATTACCGCCTTTGAGTGAGC

VF2 TGCCACCTGACGTCTAAGAA

2 


\section{Table 2 (on next page)}

List of constructed plasmids

Each plasmid backbone was constructed as an empty vector (EV) as well as with three different expression cassettes. The last two columns indicate whether the constructs were successfully transferred to and tested in either microorganism. 


\begin{tabular}{|c|c|c|c|c|}
\hline Plasmid backbone & Regulatory element & Reporter protein & E. coli & S. sp. PCC 6803 \\
\hline \multirow[t]{4}{*}{ pМJc01 } & 1 & 1 & yes & yes \\
\hline & J23101_B0032 & GFPmut3b & yes & yes \\
\hline & L21_RBS* & GFPmut3b & yes & yes \\
\hline & L21_RBS* & cystatin & yes & yes \\
\hline \multirow[t]{4}{*}{ pSB3K3 } & 1 & 1 & yes & yes \\
\hline & J23101_B0032 & GFPmut3b & yes & yes \\
\hline & L21_RBS* & GFPmut3b & yes & yes \\
\hline & L21_RBS* & cystatin & yes & yes \\
\hline \multirow[t]{4}{*}{ pPMQAK1 } & I & 1 & yes & yes \\
\hline & J23101_B0032 & GFPmut3b & yes & no \\
\hline & L21_RBS* & GFPmut3b & yes & no \\
\hline & L21_RBS* & cystatin & yes & yes \\
\hline
\end{tabular}

